\title{
Crowd Dynamics through Non-Local Conservation Laws
}

\author{
Aekta Aggarwal $^{1} \quad$ Paola Goatin $^{1}$
}

\begin{abstract}
We present a Lax-Friedrichs type scheme to compute the solutions of a class of non-local and non-linear systems of conservation laws in several space dimensions. The convergence of the approximate solutions is proved by providing suitable $\mathbf{L}^{\mathbf{1}}, \mathbf{L}^{\infty}$ and $\mathbf{B V}$ uniform bounds. To illustrate the performances of the scheme, we consider an application to crowd dynamics. Numerical integrations show the formation of lanes in groups moving in opposite directions. This is joint work with R. M. Colombo (INDAM Unit, University of Brescia).
\end{abstract}

Keywords: Nonlocal Conservation Laws, Lax-Friedrichs Scheme, Lane Formation

AMS MSC: 35L65, 65M12

\section{Introduction}

We consider the following class of non-local systems of $N$ conservation laws in 2 space dimensions:

$$
\partial_{t} U^{k}+\partial_{x} f^{k}\left(t, x, y, U^{k}, \eta * U\right)+\partial_{y} g^{k}\left(t, x, y, U^{k}, \vartheta * U\right)=0, \quad k \in\{1, \ldots, N\},
$$

where

$$
\begin{aligned}
& U(t, x, y)=\left(U^{1}, \ldots, U^{N}\right)(t, x, y) \quad \in \mathbb{R}^{N}, \quad U^{k}(t, x, y) \in \mathbb{R}, \quad k=1, \ldots, N, \\
& \eta(x, y)=\left[\begin{array}{ccc}
\eta^{11} & \ldots & \eta^{1 N} \\
\vdots & \ldots & \vdots \\
\eta^{m 1} & \ldots & \eta^{m N}
\end{array}\right](x, y) \in \mathbb{R}^{m \times N}, \quad \eta^{\ell, k}(x, y) \in \mathbb{R}, \begin{array}{l}
\ell=1, \ldots, m, \\
k=1, \ldots, N,
\end{array} \\
& \vartheta(x, y)=\left[\begin{array}{ccc}
\vartheta^{11} & \ldots & \vartheta^{1 N} \\
\vdots & \ldots & \vdots \\
\vartheta^{m 1} & \ldots & \vartheta^{m N}
\end{array}\right](x, y) \in \mathbb{R}^{m \times N}, \quad \vartheta^{\ell, k}(x, y) \in \mathbb{R}, \begin{array}{l}
\ell=1, \ldots, m, \\
k=1, \ldots, N .
\end{array}
\end{aligned}
$$

Here, $(\eta * U)(t, x, y)$ and $(\vartheta * U)(t, x, y)$ are vectors in $\mathbb{R}^{m}$ whose $\ell$-component, for $\ell=1, \ldots, m$, is

$$
\begin{aligned}
& (\eta * U)_{\ell}(t, x, y)=\iint_{\mathbb{R}^{2}} \sum_{k=1}^{N} \eta^{\ell, k}\left(x-x^{\prime}, y-y^{\prime}\right) U^{k}\left(t, x^{\prime}, y^{\prime}\right) \mathrm{d} x^{\prime} \mathrm{d} y^{\prime} \in \mathbb{R}, \\
& (\vartheta * U)_{\ell}(t, x, y)=\iint_{\mathbb{R}^{2}} \sum_{k=1}^{N} \vartheta^{\ell, k}\left(x-x^{\prime}, y-y^{\prime}\right) U^{k}\left(t, x^{\prime}, y^{\prime}\right) \mathrm{d} x^{\prime} \mathrm{d} y^{\prime} \in \mathbb{R} .
\end{aligned}
$$

In (1.1), as usual, $t$ is time, $x, y$ the space variables, $U$ the vector of the unknown densities, $\left(f^{k}, g^{k}\right)_{k=1, \ldots, N}$ the matrix valued flow and $\eta$ and $\vartheta$ are smooth convolution kernels. The coupling among the equations is only due to the non-local terms $\eta * U$ and $\vartheta * U$.

The above class of systems can model many physical phenomena such as sedimentation 3 , one-dimensional vehicular traffic [4, 7], biological applications, e.g., in structured population dynamics [13, supply chains [7, conveyor belts [11, etc. The nonlocal nature of (1.1) is particularly suitable to describe the behavior of crowds, where each individual moves according to the average

\footnotetext{
${ }^{1}$ Inria Sophia Antipolis-Méditerranée
} 
crowd density/density variation in a neighborhood of his position. The size of this neighborhood is related to the individual visual horizon. Other crowd dynamics models fitting into (1.1) were recently considered in the literature, see for example [6, 8].

Here, we use the Lax-Friedrichs type algorithm that was recently proposed in 1], generating a sequence of approximate solutions to (1.1) which, up to a subsequence, converges to a weak entropy solution of (1.1). The key point in the related convergence proof is to obtain a uniform bound on the total variation of the approximate solutions. However, a bound on the $\mathbf{L}^{\infty}$ norm and the $\mathbf{L}^{\mathbf{1}}$ Lipschitz continuous dependence of the solution on time are also obtained. These estimates allow to extend to a wider class of equations the existence results in [2, 3, 6, 7, 8. . In particular, the actual number of space dimensions is not relevant, no divergence free assumption is required, see [5], and the convolution kernels $\eta$ and $\vartheta$ are not required to have compact support.

We are interested in crowd dynamics models that capture the phenomenon of so-called lanes or paths or trails formation, which is a behavior commonly observed in the engineering literature, when two groups of people move in opposite directions. Some models were recently introduced in the mathematical literature in this regard, see [6, 8, 14. A new nonlocal model close to 8 , eq. (3.5)] is proposed in Section 3, which is shown to fit the settings of Theorem 2.2, see also [1, Theorem 2.3], and hence possesses a weak entropy solution. The numerical scheme developed in [1] is then used to compute solutions of the proposed model, showing the formation of various patterns, in particular of lanes.

The next section revisits the main results of [1, while Section 3 is devoted to sample numerical integrations of (1.1).

\section{Main Results}

Throughout, we denote $\mathbb{R}_{+}=\left[0,+\infty\left[, \mathbb{R}_{+}^{N}=\left[0,+\infty\left[^{N},|\xi|\right.\right.\right.\right.$ for a the modulus of a scalar $\xi \in \mathbb{R}$ and, for $\xi \in \mathbb{R}^{2},\|\xi\|$ stands for the usual Euclidean norm. The following assumptions on the functions $f^{k}=f^{k}(t, x, y, u, A), g^{k}=g^{k}(t, x, y, u, B)$ and $\eta, \vartheta$ in (1.1), for $k=1, \ldots, N$ are required:

(H0) $f^{k}, g^{k} \in \mathbf{C}^{2}\left(\mathbb{R}_{+} \times \mathbb{R}^{2} \times \mathbb{R} \times \mathbb{R}^{m} ; \mathbb{R}\right) ; \partial_{u} f^{k}, \partial_{u} g^{k} \in \mathbf{L}^{\infty}\left(\mathbb{R}_{+} \times \mathbb{R}^{2} \times \mathbb{R} \times \mathbb{R}^{m} ; \mathbb{R}\right)$; for all $t \in \mathbb{R}_{+}$, $(x, y) \in \mathbb{R}^{2}$ and $A, B \in \mathbb{R}^{m}, f^{k}(t, x, y, 0, A)=g^{k}(t, x, y, 0, B)=0$.

(H1) There exists a constant $M>0$ such that

$$
\begin{aligned}
& \max _{t, x, y, A}\left\{\left|\partial_{x} f^{k}\right|,\left|\partial_{x x}^{2} f^{k}\right|,\left|\partial_{x y}^{2} f^{k}\right|,\left\|\nabla_{A} f^{k}\right\|,\left\|\partial_{x} \nabla_{A} f^{k}\right\|,\left\|\partial_{y} \nabla_{A} f^{k}\right\|,\left\|\nabla_{A A}^{2} f^{k}\right\|\right\} \leq M|u|, \\
& \max _{t, x, y, B}\left\{\left|\partial_{y} g^{k}\right|,\left|\partial_{y y}^{2} g^{k}\right|,\left|\partial_{x y}^{2} g^{k}\right|,\left\|\nabla_{B} g^{k}\right\|,\left\|\partial_{x} \nabla_{B} g^{k}\right\|,\left\|\partial_{y} \nabla_{B} g^{k}\right\|,\left\|\nabla_{B B}^{2} g^{k}\right\|\right\} \leq M|u| .
\end{aligned}
$$

(H2) $\partial_{u} f^{k}, \partial_{u} g^{k} \in \mathbf{W}^{\mathbf{1}, \infty}\left(\mathbb{R}_{+} \times \mathbb{R}^{2} \times \mathbb{R} \times \mathbb{R}^{m} ; \mathbb{R}\right)$.

(H3) $\eta, \vartheta \in\left(\mathbf{C}^{2} \cap \mathbf{W}^{2, \infty}\right)\left(\mathbb{R}^{2} ; \mathbb{R}^{m \times N}\right)$.

We recall the definition of solution to the Cauchy problem for (1.1), see also [1, 3, 4, 6, 7, 8,

Definition 2.1. Let $U_{o} \in \mathbf{L}^{\infty}\left(\mathbb{R}^{2} ; \mathbb{R}^{N}\right)$. A map $U:[0, T] \rightarrow \mathbf{L}^{\infty}\left(\mathbb{R}^{2} ; \mathbb{R}^{N}\right)$ is a solution to (1.1) with initial datum $U_{o}$ if, for $k=1, \ldots, N$, setting for all $w \in \mathbb{R}$

$$
\begin{aligned}
& \tilde{f}^{k}(t, x, y, w)=f^{k}(t, x, y, w,(\eta * U)(t, x, y)), \\
& \tilde{g}^{k}(t, x, y, w)=g^{k}(t, x, y, w,(\vartheta * U)(t, x, y)),
\end{aligned}
$$

the map $U^{k}$ is a Kružkov solution to the scalar Cauchy problem

$$
\left\{\begin{array}{l}
\partial_{t} U^{k}+\partial_{x} \tilde{f}^{k}\left(t, x, y, U^{k}\right)+\partial_{y} \tilde{g}^{k}\left(t, x, y, U^{k}\right)=0 \\
U^{k}(0, x, y)=U_{o}^{k}(x, y) .
\end{array}\right.
$$


Above, by Kružkov solution we refer to [12, Definition 1]. It is immediate to observe that (H0) ensures that $U(t, x, y) \equiv 0$ solves (1.1) in the sense of Definition 2.1.

Fix a rectangular grid with mesh sizes $\Delta x$ and $\Delta y$ in $\mathbb{R}^{2}$ and a time step $\Delta t$. Also, define

$$
\begin{array}{llll}
t^{n}=n \Delta t, \quad n \in \mathbb{Z} ; & x_{i}=i \Delta x, & x_{i+1 / 2}=\left(i+\frac{1}{2}\right) \Delta x, \quad i \in \mathbb{Z} ; & \lambda_{x}=\Delta t / \Delta x, \\
y_{j}=j \Delta y, & y_{j+1 / 2}=\left(j+\frac{1}{2}\right) \Delta y, \quad j \in \mathbb{Z} ; & \lambda_{y}=\Delta t / \Delta y .
\end{array}
$$

Throughout, an initial datum $U_{o} \in\left(\mathbf{L}^{\infty} \cap \mathbf{B V}\right)\left(\mathbb{R}^{2} ; \mathbb{R}^{N}\right)$ is fixed and we denote

$$
u_{i j}^{k, 0}=\frac{1}{\Delta x \Delta y} \int_{x_{i-1 / 2}}^{x_{i+1 / 2}} \int_{y_{j-1 / 2}}^{y_{j+1 / 2}} U_{o}^{k}(x, y) \mathrm{d} x \mathrm{~d} y \quad \text { for } i, j \in \mathbb{Z}, k \in\{1, \ldots, N\} .
$$

Iteratively, we define a piecewise constant approximate solution $u_{\Delta}(t, x, y) \equiv\left(u_{\Delta}^{1}, \ldots, u_{\Delta}^{N}\right)(t, x, y)$ to (1.1) such that

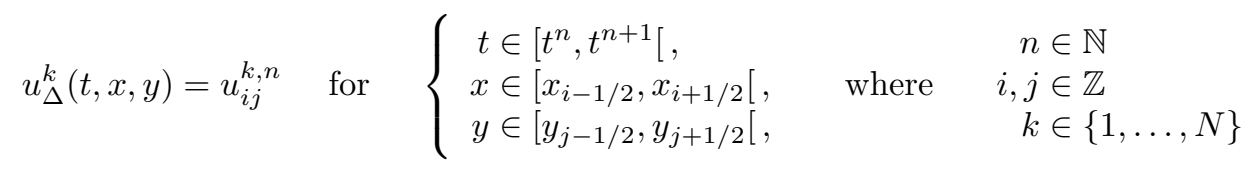

through the 5-points algorithm based on dimensional splitting, see [9, Section 3]:

$$
\begin{aligned}
& u_{i j}^{k, n+1 / 2}=u_{i j}^{k, n} \quad-\lambda_{x}\left[\quad F_{i+1 / 2, j}^{k, n}\left(u_{i j}^{k, n}, u_{i+1, j}^{k, n}\right) \quad-\quad F_{i-1 / 2, j}^{k, n}\left(u_{i-1, j}^{k, n}, u_{i j}^{k, n}\right)\right] \\
& u_{i j}^{k, n+1}=u_{i j}^{k, n+1 / 2}-\lambda_{y}\left[G_{i, j+1 / 2}^{k, n}\left(u_{i j}^{k, n+1 / 2}, u_{i, j+1}^{k, n+1 / 2}\right)-G_{i, j-1 / 2}^{k, n}\left(u_{i, j-1}^{k, n+1 / 2}, u_{i j}^{k, n+1 / 2}\right)\right]
\end{aligned}
$$

where for fixed $\alpha, \beta$ in $] 0,2 / 3[$, we define

$$
\begin{aligned}
F_{i+1 / 2, j}^{k, n}(u, v) & =\frac{f_{i+1 / 2, j}^{k, n}(u)+f_{i+1 / 2, j}^{k, n}(v)}{2}-\frac{\alpha(v-u)}{2 \lambda_{x}}, \\
f_{i+1 / 2, j}^{k, n}(u) & =f^{k}\left(t^{n}, x_{i+1 / 2}, y_{j}, u, A_{i+1 / 2, j}^{n}\right), \\
G_{i, j+1 / 2}^{k, n}(u, v) & =\frac{g_{i, j+1 / 2}^{k, n+1 / 2}(u)+g_{i, j+1 / 2}^{k, n+1 / 2}(v)}{2}-\frac{\beta(v-u)}{2 \lambda_{y}}, \\
g_{i, j+1 / 2}^{k, n+1 / 2}(u) & =g^{k}\left(t^{n+1 / 2}, x_{i}, y_{j+1 / 2}, u, B_{i, j+1 / 2}^{n+1 / 2}\right) .
\end{aligned}
$$

The convolution terms are computed through quadrature formulæ, so that they are given by

$$
\begin{aligned}
& A_{i+\frac{1}{2}, j}^{n}=\Delta x \Delta y\left[\sum_{l, p \in \mathbb{Z}} \sum_{k=1}^{N} \eta_{i+1 / 2-l, j-p}^{1, k} u_{l+1 / 2, p}^{k, n}, \ldots, \sum_{l, p \in \mathbb{Z}} \sum_{k=1}^{N} \eta_{i+1 / 2-l, j-p}^{m, k} u_{l+1 / 2, p}^{k, n}\right] \\
& B_{i, j+\frac{1}{2}}^{n+1 / 2}=\Delta x \Delta y\left[\sum_{l, p \in \mathbb{Z}} \sum_{k=1}^{N} \vartheta_{i-l, j+1 / 2-p}^{1, k} u_{l, p+1 / 2}^{k, n+1 / 2}, \ldots, \sum_{l, p \in \mathbb{Z}} \sum_{k=1}^{N} \vartheta_{i-l, j+1 / 2-p}^{m, k} u_{l, p+1 / 2}^{k, n+1 / 2}\right]
\end{aligned}
$$

where, for instance, $u_{l+1 / 2, p}^{k, n}$ is a convex combination of $u_{l, p}^{k, n}$ and $u_{l+1, p}^{k, n}$, moreover

$$
\eta_{i+1 / 2, j}^{\ell, k}=\eta^{\ell, k}\left(x_{i+1 / 2}, y_{j}\right) \quad \text { and } \quad \vartheta_{i, j+1 / 2}^{\ell, k}=\vartheta^{\ell, k}\left(x_{i}, y_{j+1 / 2}\right) .
$$

Throughout, $\Delta t$ must satisfy the CFL condition

$$
\lambda_{x} \leq \frac{\min \{6 \alpha, 4-6 \alpha, 1\}}{1+6 \max _{k}\left\|\partial_{u} f^{k}\right\|_{\mathbf{L}^{\infty}}}, \quad \lambda_{y} \leq \frac{\min \{6 \beta, 4-6 \beta, 1\}}{1+6 \max _{k}\left\|\partial_{u} g^{k}\right\|_{\mathbf{L}^{\infty}}},
$$

and we assume that the mesh sizes are sufficiently small, in the sense that $\Delta x, \Delta y \leq 1 /(3 M)$, where $M$ is as in (H1). The main result in [1] is as follows: 
Theorem 2.2. Let assumptions (HO), (H1), (H2), (H3) and conditions (2.5) hold. Fix an initial datum $U_{o} \in\left(\mathbf{L}^{\mathbf{1}} \cap \mathbf{L}^{\infty} \cap \mathbf{B V}\right)\left(\mathbb{R}^{2} ; \mathbb{R}_{+}^{N}\right)$. Then, the algorithm (2.2) defines a sequence of approximate solutions which converges, up to a subsequence, to a solution $U \in \mathbf{C}^{\mathbf{0}}\left(\mathbb{R}_{+} ; \mathbf{L}^{\mathbf{1}}\left(\mathbb{R}^{2} ; \mathbb{R}_{+}^{N}\right)\right)$ of (1.1) in the sense of Definition 2.1. Moreover, $U$ is locally Lipschitz continuous with respect to the $\mathbf{L}^{\mathbf{1}}$ norm and for all $k \in\{1, \ldots, N\}$ and for all $t \in \mathbb{R}_{+}$, the following bounds hold:

$$
\begin{aligned}
& \|U(t)\|_{\mathbf{L}^{\infty}\left(\mathbb{R}^{2} ; \mathbb{R}^{N}\right)} \leq e^{\mathcal{C} t\left(1+\left\|U_{o}\right\|_{\mathbf{L}^{1}}\right)}\left\|U_{o}\right\|_{\mathbf{L}^{\infty}\left(\mathbb{R}^{2} ; \mathbb{R}^{N}\right)}, \\
& \left\|U^{k}(t)\right\|_{\mathbf{L}^{1}\left(\mathbb{R}^{2} ; \mathbb{R}\right)}=\left\|U_{o}^{k}\right\|_{\mathbf{L}^{1}\left(\mathbb{R}^{2} ; \mathbb{R}\right)}, \\
& \operatorname{TV}\left(U^{k}(t)\right) \leq e^{\mathcal{K}_{1} t} \operatorname{TV}\left(U_{o}^{k}\right)+\mathcal{K}_{2}\left(e^{\mathcal{K}_{1} t}-1\right), \\
& \|U(t+\tau)-U(t)\|_{\mathbf{L}^{1}\left(\mathbb{R}^{2} ; \mathbb{R}^{N}\right)} \leq C(t) \tau,
\end{aligned}
$$

where $\mathcal{C}, \mathcal{K}_{1}, \mathcal{K}_{2}$ and $C(t)$ are constants defined in Lemma2.3 below.

The proof is based on Lemma 2.3 stated below. In view of [1, Remark 2.1], (H0) and (H3), Theorem 2.2 ensures the existence of a solution and the validity of the bounds above on any finite time interval, whenever the initial datum is compactly supported.

Lemma 2.3. Let assumptions (HO), (H1), (H2), (H3) and condition (2.5) hold. Fix $U_{o} \in$ $\left(\mathbf{L}^{1} \cap \mathbf{L}^{\infty} \cap \mathbf{B V}\right)\left(\mathbb{R}^{2} ; \mathbb{R}_{+}^{N}\right)$. Then, the approximate solution $u_{\Delta}$ defined by the algorithm (2.2) satisfies

(a) (Positivity) $u_{\Delta}^{k}(t, x, y) \geq 0$ for all $k \in\{1, \ldots, N\}, t \in \mathbb{R}_{+}$and $(x, y) \in \mathbb{R}^{2}$.

(b) $\left(\mathbf{L}^{\mathbf{1}}\right.$ bound) $\left\|u_{\Delta}^{k}(t)\right\|_{\mathbf{L}^{\mathbf{1}}}=\left\|u_{\Delta}^{k}(0)\right\|_{\mathbf{L}^{1}}$ for all $k \in\{1, \ldots, N\}$ and $t \in \mathbb{R}_{+}$.

(c) $\left(\mathbf{L}^{\infty}\right.$ bound) For all $t \in \mathbb{R}_{+}$,

$$
\left\|u_{\Delta}(t)\right\|_{\mathbf{L}^{\infty}} \leq e^{\mathcal{C} t\left(1+\left\|U_{o}\right\|_{\mathbf{L}^{1}}\right)}\left\|U_{o}\right\|_{\mathbf{L}^{\infty}}
$$

where $\mathcal{C}$ depends only on $\eta, \vartheta, f^{1}, \ldots, f^{N}, g^{1}, \ldots, g^{N}$.

(d) (BV bound) For all $n$, for all $t \in\left[t^{n}, t^{n+1}[\right.$ and for all $k=1, \ldots, N$

$$
\begin{aligned}
& \sum_{i j}\left(\left|u_{i+1, j}^{k, n}-u_{i j}^{k, n}\right| \Delta y+\left|u_{i, j+1}^{k, n}-u_{i j}^{k, n}\right| \Delta x\right) \\
\leq & e^{\mathcal{K}_{1} t} \sum_{i j}\left(\left|u_{i+1, j}^{k, 0}-u_{i j}^{k, 0}\right| \Delta y+\left|u_{i, j+1}^{k, 0}-u_{i j}^{k, 0}\right| \Delta x\right)+\mathcal{K}_{2}\left(e^{\mathcal{K}_{1} t}-1\right) .
\end{aligned}
$$

where $\mathcal{K}_{1}$ and $\mathcal{K}_{2}$ are constants depending on $\left\|U_{o}\right\|_{\mathbf{L}^{1}}$ and on the functions $\eta, \vartheta, f^{k}$ and $g^{k}$.

(e) (Discrete entropy condition) For all $i, j \in \mathbb{Z}$, for all $k=1, \ldots, N$ and for all $\kappa \in \mathbb{R}$,

$$
\begin{aligned}
\left|u_{i j}^{k, n+1}-\kappa\right| & -\left|u_{i j}^{k, n}-\kappa\right|+\lambda_{x}\left(\Phi_{i+1 / 2, j}^{k, n, \kappa}\left(u_{i j}^{k, n}, u_{i+1, j}^{k, n}\right)-\Phi_{i-1 / 2, j}^{k, n, \kappa}\left(u_{i-1, j}^{k, n}, u_{i j}^{k, n}\right)\right) \\
& +\lambda_{x} \operatorname{sgn}\left(u_{i j}^{k, n+1 / 2}-\kappa\right)\left(f_{i+1 / 2, j}^{k, n}(\kappa)-f_{i-1 / 2, j}^{k, n}(\kappa)\right) \\
& +\lambda_{y}\left(\Gamma_{i, j+1 / 2}^{k, n, \kappa}\left(u_{i j}^{k, n+1 / 2}, u_{i, j+1}^{k, n+1 / 2}\right)-\Gamma_{i, j-1 / 2}^{k, n, \kappa}\left(u_{i, j-1}^{k, n+1 / 2}, u_{i j}^{k, n+1 / 2}\right)\right) \\
& +\lambda_{y} \operatorname{sgn}\left(u_{i j}^{k, n+1}-\kappa\right)\left(g_{i, j+1 / 2}^{k, n+1 / 2}(\kappa)-g_{i, j-1 / 2}^{k, n+1 / 2}(\kappa)\right)
\end{aligned}
$$

where $\Phi$ and $\Gamma$ are the Kružkov numerical entropy flows, see [2, Proposition 2.8], [9, Section 3], [10, Section 4], given by

$$
\begin{aligned}
\Phi_{i+1 / 2, j}^{k, n, \kappa}\left(u_{1}, u_{2}\right) & =f_{i+1 / 2, j}^{k, n}\left(u_{1} \vee \kappa, u_{2} \vee \kappa\right)-f_{i+1 / 2, j}^{k, n}\left(u_{1} \wedge \kappa, u_{2} \wedge \kappa\right), \\
\Gamma_{i, j+1 / 2}^{k, n, \kappa}\left(u_{1}, u_{2}\right) & =g_{i, j+1 / 2}^{k, n+1 / 2}\left(u_{1} \vee \kappa, u_{2} \vee \kappa\right)-g_{i, j+1 / 2}^{k, n+1 / 2}\left(u_{1} \wedge \kappa, u_{2} \wedge \kappa\right) .
\end{aligned}
$$


(f) ( $\mathbf{L}^{1}$ Lipschitz Continuity in time) For all $n=1, \ldots, \bar{n}, \bar{n} \in \mathbb{N}$ fixed,

$$
\left\|u_{\Delta}\left(t^{n+1}\right)-u_{\Delta}\left(t^{n}\right)\right\|_{\mathbf{L}^{1}} \leq C \Delta t .
$$

where $C$ is a constant depending on $\left\|U_{o}\right\|_{\mathrm{L}^{1}}, \mathrm{TV}\left(U_{o}\right), t^{n}, \lambda_{x}, \lambda_{y} \alpha, \beta$ and on the functions $\eta, \vartheta, f^{k}$ and $g^{k}$, for $k \in\{1, \ldots, N\}$.

For the proofs of Theorem 2.2 and Lemma 2.3, refer to 1 .

\section{$3 \quad$ Numerical Integrations}

We refer to the class of nonlocal crowd dynamics models introduced in [8], for two populations trying to avoid each other and described by their densities $U^{1}, U^{2}$. In particular, we consider

$$
\left\{\begin{array}{l}
\partial_{t} U^{1}+\operatorname{div}\left(c_{1} U^{1}\left(1-U^{1}\right)\left(\left(1-\varepsilon_{1} \frac{U^{1} * \mu}{\sqrt{1+\left\|U^{1} * \mu\right\|^{2}}}\right) \vec{v}^{1}(x, y)-\varepsilon_{2} \frac{\nabla U^{2} * \mu}{\sqrt{1+\left\|\nabla U^{2} * \mu\right\|^{2}}}\right)\right)=0, \\
\partial_{t} U^{2}+\operatorname{div}\left(c_{2} U^{2}\left(1-U^{2}\right)\left(\left(1-\varepsilon_{1} \frac{U^{2} * \mu}{\sqrt{1+\left\|U^{2} * \mu\right\|^{2}}}\right) \vec{v}^{2}(x, y)-\varepsilon_{2} \frac{\nabla U^{1} * \mu}{\sqrt{1+\left\|\nabla U^{1} * \mu\right\|^{2}}}\right)\right)=0 .
\end{array}\right.
$$

For $i, j=1,2, j \neq i, c_{i} U^{i}\left(1-U^{i}\right), c_{i}>0$ is the crowding factor and $\vec{v}^{i}(x, y)=\left[v_{i 1}, v_{i 2}\right](x, y)$ defines the preferred path for $i$-th population where each pedestrian moves in the preferred direction $\vec{v}^{i}(x, y)$ but scales his speed according to $\left(1-\varepsilon_{i}\left(U^{i} * \mu\right) / \sqrt{1+\left\|U^{i} * \mu\right\|^{2}}\right)$, i.e., according to the density of its own population, which he sees in his horizon. The $i$-th population also deviates from its preferred trajectory due to the interaction with the individuals of the $j$-th population according to $-\varepsilon_{j}\left(\nabla U^{j} * \mu\right) / \sqrt{1+\left\|\nabla U^{j} * \mu\right\|^{2}}$.

Lemma 3.1. System (3.1) fits into (1.1) setting, $N=2, m=6$ and

$$
\begin{aligned}
& f(t, x, y, U, A)=\left[\begin{array}{c}
c_{1} U^{1}\left(1-U^{1}\right)\left(\left(1-\varepsilon_{1} \frac{A_{1}}{\sqrt{1+A_{1}^{2}}}\right) v_{11}(x, y)-\varepsilon_{2} \frac{A_{5}}{\sqrt{1+A_{5}{ }^{2}+A_{6}{ }^{2}}}\right) \\
c_{2} U^{2}\left(1-U^{2}\right)\left(\left(1-\varepsilon_{1} \frac{A_{4}}{\sqrt{1+A_{4}^{2}}}\right) v_{21}(x, y)-\varepsilon_{2} \frac{A_{2}}{\sqrt{1+A_{2}{ }^{2}+A_{3}{ }^{2}}}\right)
\end{array}\right], \\
& g(t, x, y, U, B)=\left[\begin{array}{c}
c_{1} U^{1}\left(1-U^{1}\right)\left(\left(1-\varepsilon_{1} \frac{B_{1}}{\sqrt{1+B_{1}^{2}}}\right) v_{12}(x, y)-\varepsilon_{2} \frac{B_{6}}{\sqrt{1+B_{5}^{2}+B_{6}^{2}}}\right) \\
c_{2} U^{2}\left(1-U^{2}\right)\left(\left(1-\varepsilon_{1} \frac{B_{4}}{\sqrt{1+B_{4}^{2}}}\right) v_{22}(x, y)-\varepsilon_{2} \frac{B_{3}}{\sqrt{1+B_{2}{ }^{2}+B_{3}^{2}}}\right)
\end{array}\right],
\end{aligned}
$$

and

$$
\eta=\vartheta=\left[\begin{array}{cccccc}
\mu & \partial_{x} \mu & \partial_{y} \mu & 0 & 0 & 0 \\
0 & 0 & 0 & \mu & \partial_{x} \mu & \partial_{y} \mu
\end{array}\right]^{T},
$$

with $A=\eta * U, B=\vartheta * U, A, B \in \mathbb{R}^{6}$. Moreover, if $\vec{v}^{i} \in\left(\mathbf{C}^{2} \cap \mathbf{W}^{2, \infty}\right)\left(\mathbb{R}^{2} ; \mathbb{R}^{2}\right)$ and $\mu \in\left(\mathbf{C}^{\mathbf{3}} \cap\right.$ $\left.\mathbf{W}^{\mathbf{3}, \infty}\right)\left(\mathbb{R}^{2} ; \mathbb{R}\right)$, for any compactly supported initial datum $\left(U_{o}^{1}, U_{o}^{2}\right) \in\left(\mathbf{L}^{\mathbf{1}} \cap \mathbf{L}^{\infty} \cap \mathbf{B V}\right)\left(\mathbb{R}^{2} ;[0,1]^{2}\right)$ problem (3.1) admits a solution defined for all $t \in \mathbb{R}_{+}$and satisfying the estimates in Theorem [2.2.

The proof is immediate and follows the same lines of [1, Lemma 3.1]. Two different situations are considered and numerical integrations obtained with the algorithm described in Section 2 are exhibited. By Definition 2.1] [12, Theorem 3], the choices (3.5) and (3.9) of the initial data, the maximal density of solutions can not exceed 1 , which is consistent with the results of the integrations. 


\subsection{Two Crowds Moving Radially in Opposite Directions}

In the domain $[-4,4] \times[-4,4]$, two groups of people, one concentrated in a circle of radius $\sqrt{4.3}$ at the origin and other concentrated in an annulus between concentric circles (centered at origin) of radii $\sqrt{4.5}$ and $\sqrt{8.8}$, move radially outward and inward respectively. Thus, in (3.1) we choose:

$$
\begin{aligned}
c_{1}=c_{2} & =4, \quad \varepsilon_{1}=0.3, \quad \varepsilon_{2}=0.7 \\
\vec{v}^{1}(x, y) & =-\vec{v}^{2}(x, y)=\frac{[x, y]}{1+x^{2}+y^{2}}, \\
\mu(x, y) & =\left(\left(1-4 x^{2}\right)\left(1-4 y^{2}\right)\right)^{3} \chi_{[-0.5,0.5]^{2}}(x, y), \quad \tilde{\mu}(x, y)=\frac{1}{\iint_{\mathbb{R}^{2}} \tilde{\mu}} \tilde{\mu}(x, y), \\
{\left[\begin{array}{c}
U_{o}^{1} \\
U_{o}^{2}
\end{array}\right](x, y) } & =0.9\left[\begin{array}{l}
\chi_{\left\{(x, y): x^{2}+y^{2} \leq 4.3\right\}} \\
\chi_{\left\{(x, y): 4.5 \leq x^{2}+y^{2} \leq 8.8\right\}}
\end{array}\right](x, y) .
\end{aligned}
$$

Figure 1 shows the $\mathbf{L}^{\mathbf{1}}$ norm of the solution $\left(U_{1}, U_{2}\right)$ as a function of time $t$. It can be seen that the

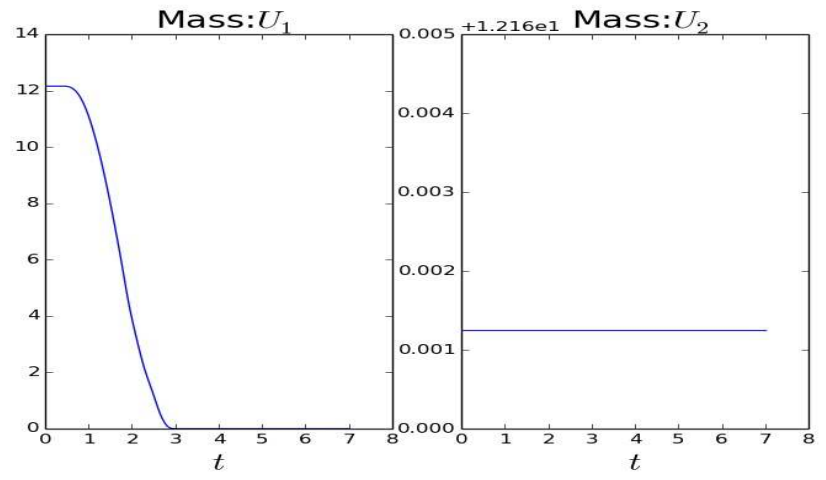

Figure 1: Time evolution of the $\mathbf{L}^{\mathbf{1}}$ norm of $\left(U_{1}, U_{2}\right)$ as a function of time $t$.

mass of the first crowd decreases to zero, while the mass of second crowd remains constant. Evident pattern formation can be observed on numerical integration of (3.1)-(3.5), shown in Figure 2 As expected, the first crowd moves radially outward and the second one radially inward. The high density regions of one crowd are very efficiently complemented by low density regions of the other group. Symmetric lane formation creates paths through which the other group can move. After some time, the first group exits the domain, though the second group takes longer time to settle to a perfect circular shape.

\subsection{A Moving Crowd Interacting with a Standing Crowd}

Consider two groups of people in the domain $[-1.7,4.2] \times[-1.7,1.7]$ with a single exit at $x=4.2$. At time 0 , one group represented by $U_{1}$ starts moving towards the exit while the other group, $U_{2}$, wants to stay and moves only as a reaction to the exiting population. The space available to the pedestrians is the rectangle $[-1.4,4.2] \times[-1.4,1.4]$ due to the presence of the walls of 0.3 width along $\{-1.7\} \times[-1.7,1.7]$, and $[-1.7,4.2] \times\{-1.7\}$ and $[-1.7,4.2] \times\{1.7\}$.

As in [8], the preferred path of each pedestrian is given by the sum $g+\delta$. The vector $g$ is tangent to the geodesic towards the pedestrian's target or 0 when pedestrians would not move. The vector $\delta$ describes the discomfort of pedestrians when walking close to a wall. It is perpendicular to the walls and pointing inward. Its maximum modulus is $\delta_{\max }=0.8$ at walls and decreases to zero at a distance $\delta_{r}=0.6$ from walls. As indicated earlier, both crowds scale their speed due to their own density and deviate from their preferred trajectory due the presence of the other group present in their horizon. In (3.1) we choose:

$$
c_{1}=2, c_{2}=1, \quad \varepsilon_{1}=0.3, \quad \varepsilon_{2}=0.7
$$



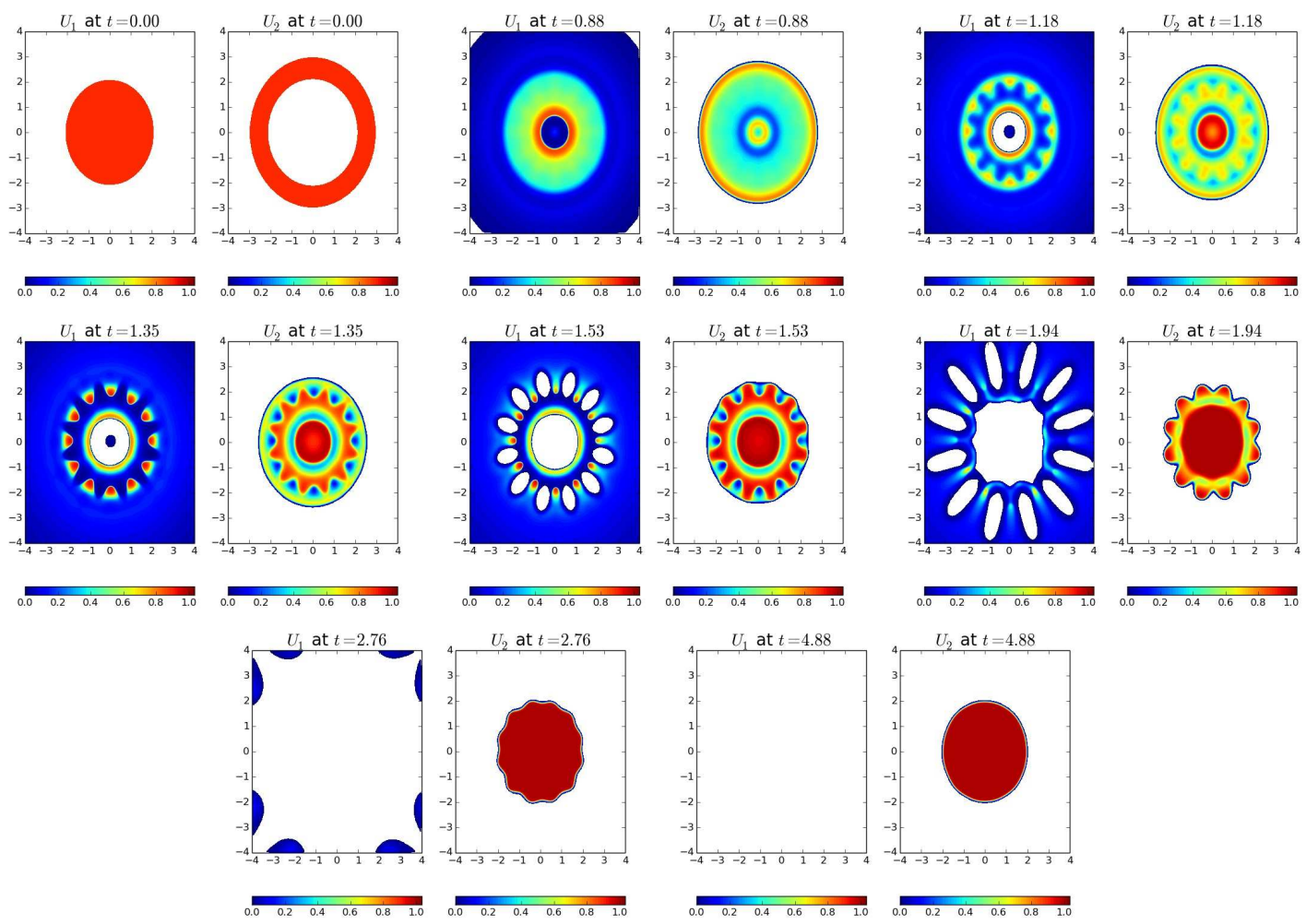

Figure 2: Solution to (3.1) - 3.5) with space mesh with $\Delta x=\Delta y=.0125, \lambda_{x}=\lambda_{y}=.05$, $\Delta t=6.25 E-4$ and $\alpha=\beta=.3333$. A symmetric(radial) formation of lanes is noticed for both the crowds. Each crowd forms lanes to pave paths for the other crowd to move in/out of the domain. The lanes formed by the two crowds are complimentary to each other. The formation of the lanes is noticed till the first crowd $U_{1}$ completely exits the domain.

$$
\begin{aligned}
& {\left[\vec{v}^{1} ; \vec{v}^{2}\right](x, y) }=[[1,0]+\delta ;[0,0]+\delta] \\
& \mu(x, y)=\left(\left(1-16 x^{2}\right)\left(1-16 y^{2}\right)\right)^{3} \chi_{[-0.25,0.25]^{2}}(x, y), \quad \tilde{\mu}(x, y)=\frac{1}{\iint_{\mathbb{R}^{2}} \tilde{\mu}} \tilde{\mu}(x, y), \\
& {\left[\begin{array}{c}
U_{o}^{1} \\
U_{o}^{2}
\end{array}\right](x, y)=\left[\begin{array}{c}
0.9 \chi_{\left\{(x, y): x^{2}+y^{2} \leq 1, y \geq 0\right\}}+0.7 \chi_{\left\{(x, y): x^{2}+y^{2} \leq 1, y \leq 0\right\}} \\
0.7 \chi_{\left\{(x, y): x^{2}+y^{2} \leq 1, y \geq 0\right\}}+0.9 \chi_{\left\{(x, y): x^{2}+y^{2} \leq 1, y \leq 0\right\}}
\end{array}\right](x, y) . }
\end{aligned}
$$

The numerical integration of 3.1 3.9 is shown in Figure 3. The first group moves to the right. The second crowd, though prefers not to move, moves and forms lanes to pave the path for the first crowd to move. After the first crowd has completely vacated the room, the density of the second crowd keeps moving for a while.

Figure 4 shows the $\mathbf{L}^{\mathbf{1}}$ norm of the solution $\left(U_{1}, U_{2}\right)$ as a function of time $t$. It can be seen that the mass of the first crowd decreases to zero, while the mass of second crowd, remains constant.

Acknowledgements. This work was partially supported by the European Research Council under the European Union's Seventh Framework Program (FP/2007-2013) ERC Grant Agreement n. 257661. The authors would like to thank the third co-author R. M. Colombo (INDAM Unit, University of Brescia) who contributed significantly to this work. 



Figure 3: Solution to (3.1)-(3.9) computed with $\Delta x=\Delta y=.01, \lambda_{x}=\lambda_{y}=.05, \Delta t=.005$ and $\alpha=\beta=.3333$. Note that though the second crowd $U_{2}$ does not prefer to move, the model makes $U_{2}$ to adjust and develop lanes to allow the first crowd $U_{1}$ to move along its preferred path to right. The lane formation ceases to exist after the first crowd $U_{1}$ has completely vacated the domain.

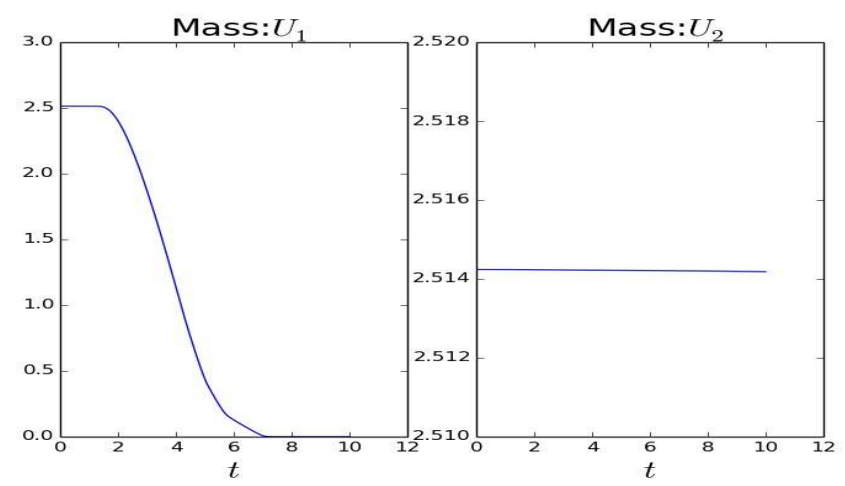

Figure 4: Time evolution of the $\mathbf{L}^{1}$ norm of $\left(U_{1}, U_{2}\right)$ as a function of $t$.

\section{References}

[1] A. Aggarwal, R. M. Colombo, and P. Goatin. Nonlocal systems of conservation laws in several space dimensions. SIAM Journal on Numerical Analysis, 53(2):963-983, 2015.

[2] P. Amorim, R. M. Colombo, and A. Teixeira. On the numerical integration of scalar nonlocal conservation laws. ESAIM M2AN, 49(1):19-37, 2015.

[3] F. Betancourt, R. Bürger, K. H. Karlsen, and E. M. Tory. On nonlocal conservation laws modelling sedimentation. Nonlinearity, 24(3):855-885, 2011. 
[4] S. Blandin and P. Goatin. Well-posedness of a conservation law with non-local flux arising in traffic flow modeling. Numerische Mathematik, pages 1-25, 2015.

[5] C. Chainais-Hillairet. Finite volume schemes for a nonlinear hyperbolic equation. Convergence towards the entropy solution and error estimate. M2AN Math. Model. Numer. Anal., 33(1):129-156, 1999.

[6] R. M. Colombo, M. Garavello, and M. Lécureux-Mercier. A class of nonlocal models for pedestrian traffic. Math. Models Methods Appl. Sci., 22(4):1150023, 34, 2012.

[7] R. M. Colombo, M. Herty, and M. Mercier. Control of the continuity equation with a non local flow. ESAIM Control Optim. Calc. Var., 17(2):353-379, 2011.

[8] R. M. Colombo and L.-M. Mercier. Nonlocal crowd dynamics models for several populations. Acta Mathematica Scientia, 32(1):177-196, 2011.

[9] M. Crandall and A. Majda. The method of fractional steps for conservation laws. Numer. Math., 34(3):285-314, 1980.

[10] M. G. Crandall and A. Majda. Monotone difference approximations for scalar conservation laws. Math. Comp., 34(149):1-21, 1980.

[11] S. Göttlich, S. Hoher, P. Schindler, V. Schleper, and A. Verl. Modeling, simulation and validation of material flow on conveyor belts. Appl. Math. Mod., 38(13):3295 - 3313, 2014.

[12] S. N. Kružhkov. First order quasilinear equations with several independent variables. Mat. Sb. (N.S.), 81 (123):228-255, 1970.

[13] B. Perthame. Transport equations in biology. Frontiers in Mathematics. Birkhäuser Verlag, Basel, 2007.

[14] E. Tory, H. Schwandt, R. Ruiz-Baier, and S. Berres. An adaptive finite-volume method for a model of two-phase pedestrian flow. Networks and Heterogeneous Media, 6(3):401-423, 2011. 